\title{
$\delta$-Sunspot Formation in Simulation of Active-Region-Scale Flux Emergence
}

\author{
Fang Fang, Yuhong Fan \\ High Altitude Observatory, National Center for Atmospheric Research, 3090 Center Green Dr, \\ Boulder, CO 80301
}

\begin{abstract}
$\delta$-sunspots, with highly complex magnetic structures, are very productive in energetic eruptive events, such as X-class flares and homologous eruptions. We here study the formation of such complex magnetic structures by numerical simulations of magnetic flux emergence from the convection zone into the corona in an active-region-scale domain. In our simulation, two pairs of bipolar sunspots form on the surface, originating from two buoyant segments of a single subsurface twisted flux rope, following the approach of Toriumi et al. (2014). Expansion and rotation of the emerging fields in the two bipoles drive the two opposite polarities into each other with apparent rotating motion, producing a compact $\delta$-sunspot with a sharp polarity inversion line. The formation of the $\delta$-sunspot in such a realistic-scale domain produces emerging patterns similar to those formed in observations, e.g. the inverted polarity against Hale's law, the curvilinear motion of the spot, strong transverse field with highly sheared magnetic and velocity fields at the PIL. Strong current builds up at the PIL, giving rise to reconnection, which produces a complex coronal magnetic connectivity with non-potential fields in the $\delta$-spot overlaid by more relaxed fields connecting the two polarities at the two ends.
\end{abstract}

Subject headings: magnetohydrodynamics(MHD) — Sun: sunspots — Sun: photosphere

\section{Introduction}

$\delta$-sunspots, where two spots of opposite polarities share the same penumbrae, are of particular interest due to their high productivity of powerful flares and coronal mass ejections (CMEs) (Hagvard et al. 1984; Tanaka 1991; Schmieder et al. 1994; Sammis et al. 2000; Liu \& Zhang 2001). Based on their characteristics during the formation observed at Big Bear Solar Observatory, Zirin \& Liggett (1987) categorize the $\delta$-sunspots into three types: island $\delta$ which emerge at once but with different dipoles intertwined, single $\delta$ with large satellite dipoles emerging in the penumbra of a large spot, and a third and most popular type formed by collision of two bipolar spot groups. Regardless of their types, all the $\delta$ spots, which form from opposite polarities of different dipoles and die together, are short-lived complex structures with highly sheared field lines at the 
PIL. They manifests their complexity on the photosphere as highly structured active regions, with non-potential coronal fields in the form of sheared and stretched field lines connecting the sunspots (Patty \& Hagyard 1986; Tanaka 1991; Lites et al. 1995; Leka 1997), storing a large amount of free magnetic energy available for the flare activities. In many occasions, the footpoints of the $\delta$-spots exhibit shearing motion at the PIL and rotation of the spots (Tanaka 1991; Leka et al. 1996), which further stretch and twist the magnetic field lines. The combination of non-potential magnetic field and shearing, rotating motion at the PIL produces a magnetic configuration that is prone to magnetic reconnection and eruption, which might be repetitive in the presence of energizing motions (Fang et al. 2012a).

According to the observed features of compact and complex $\delta$-spots regions, Zirin \& Liggett (1987) point out that they may be the result of a subsurface phenomenon which locks the two spots closely together and it is therefore important to investigate the subsurface processes that form and maintain these complex spots and build up the free energy. Later Tanaka (1991) and Leka et al. (1996) suggest that $\delta$-sunspots can be formed by the emergence of highly twisted magnetic flux tubes from the convection zone, which can explain self-consistently the shearing and rotation motion as well as the compact pattern of opposite polarities. This concept then motivated series of numerical simulations on helical kink instability of highly twisted rising flux tubes emerging from the interior (Linton et al. 1998, 1999; Fan et al. 1999). The kinked evolution of the rising tubes produces a compact magnetic bipolar region, with polarity order reversed from Hale's law, with great similarities to observed features of $\delta$-sunspots. The braided kinked magnetic structure keeps the two opposite polarities closely together and drives the rotation around each other. Tanaka (1991) also proposed a model which involved the successive emergences of two twisted magnetic flux ropes which may or may not be interconnected under the surface, to explain the evolution of complex $\delta$-spots. Recent study on the quadrupolar active region 11158 with colliding $\delta$-spots (Sun et al. 2012) found that the magnetic fields were highly sheared at the PIL carrying strong current, and significant free energy was injected into the atmosphere. Later Toriumi et al. (2014) considered two scenarios of the subsurface emerging structures for simulating the formation of $\delta$-spots: two flux ropes each with one buoyant section, and a single flux rope with two buoyant sections. They found that only the emergence of the two buoyant sections from a single flux rope forms a sharp PIL in the domain. Following this work, here we also carry out a simulation of the emergence of a twisted flux rope with two buoyantly rising sections, but in a domain of size comparable to active regions to study whether compressed $\delta$-spot like structures of realistic active region size scales can still form. In the following sections, we describe the numerical setup of our simulation in Section 2 , and investigate the formation of $\delta$-sunpots and the photospheric flows and energy flux in Section 3 and 4, respectively, followed by conclusions in Section 5 . 


\section{Simulation Setup}

We solve the ideal MHD equations within the Block Adaptive Tree Solar-wind Roe Upwind Scheme (BATS-R-US) that was developed at the University of Michigan (Powell et al. 1999; Tóth et al. 2012):

$$
\begin{gathered}
\frac{\partial \rho}{\partial t}+\nabla \cdot(\rho \mathbf{u})=0 \\
\frac{\partial(\rho \mathbf{u})}{\partial t}+\nabla \cdot\left[\rho \mathbf{u u}+\left(p+\frac{\mathbf{B B}}{8 \pi}\right) \mathbf{I}-\frac{\mathbf{B B}}{4 \pi}\right]=\rho \mathbf{g} \\
\frac{\partial E}{\partial t}+\nabla \cdot\left[\left(E+p+\frac{\mathbf{B} \cdot \mathbf{B}}{8 \pi}\right) \mathbf{u}-\frac{(\mathbf{u} \cdot \mathbf{B}) \mathbf{B}}{4 \pi}\right]=0, \\
\frac{\partial \mathbf{B}}{\partial t}=\nabla \times(\mathbf{u} \times \mathbf{B})
\end{gathered}
$$

where $\rho, \mathbf{u}, E, p, T, \mathbf{B}$ and $\mathbf{g}$ are the density, velocity, total energy density, pressure, temperature, magnetic field and gravitational acceleration, respectively. The domain of our simulation covers $158(\mathrm{X}) \times 138(\mathrm{Y}) \times 79(\mathrm{Z}) \mathrm{Mm}^{3}$, extending $20 \mathrm{Mm}$ below the photosphere and $59 \mathrm{Mm}$ into the corona with an initial background stratification of atmosphere in hydrostatic equilibrium (Fan 2001). The cell sizes range from 38.6 to $618.68 \mathrm{~km}$ in the vertical direction from the interior to coronal height and 77.3 to $1237.4 \mathrm{~km}$ in horizontal directions. A horizontal flux rope in the $\mathbf{x}$ direction is inserted

at depth $\mathrm{Z}=-10 \mathrm{Mm}$, with the magnetic field given by $\mathbf{B}=B_{0} e^{-r^{2} / a^{2}} \hat{\boldsymbol{x}}+q r B_{0} e^{-r^{2} / a^{2}} \hat{\boldsymbol{\theta}}$, where $B_{0}=-12 \mathrm{kG}$, is the strength of the magnetic fields at the central axis of the rope, $\hat{\boldsymbol{\theta}}$ is the azimuthal direction in the cross section of the flux rope, $q=0.50 / a$, is the rate of twist per unit length, $a=3.0 \mathrm{Mm}$ is the radius of Gaussian decay, and $r$ is the distance from the axis of the flux rope. Fig. [1] shows the vertical stratification of pressure and temperature in the domain. The green line represents the distribution of magnetic energy inside the flux rope in a cross-section cut. The plasma beta at the central axis of the flux rope, $\beta=66$. The initial rope is density-depleted at two segments centered at $x= \pm 15 \mathrm{Mm}$ as shown in Fig. 2, with perturbation as:

$$
\Delta \rho=-\frac{0.5\left[B_{0} e^{\left(-r^{2} / a^{2}\right)}\right]^{2}}{p} \rho_{0} \begin{cases}-\eta+\frac{(1+\eta) \cos \left[\pi\left(x-x_{+}\right) / \lambda\right]}{2}, & \text { if }\left|x-x_{+}\right| \leq \lambda \\ -\eta+\frac{(1+\eta) \cos \left[\pi\left(x-x_{-}\right) / \lambda\right]}{2}, & \text { if }\left|x-x_{-}\right| \leq \lambda \\ -\eta, & \text { otherwise. }\end{cases}
$$

Here $\rho_{0}$ is the initial density value in hydrostatic equilibrium, buoyancy factor $\eta=0.15$, and $\mathrm{x}_{ \pm}$ $= \pm 15 \mathrm{Mm}, \lambda=4.6 \mathrm{Mm}$ is the half length of each buoyant segments. And the thermal pressure inside the flux rope is modified to maintain the total pressure balance.

\section{Formation of $\delta$-sunspots}

The density-depleted sections, centered at $\mathrm{x}= \pm 15 \mathrm{Mm}$ of the initial flux rope, rise in the convection zone under buoyancy. The dramatic decrease of pressure in the ambient plasma with 
altitude gives rise to the vast expansion in the two rising sections of the flux rope as it emerges. Fig. 3 illustrates the three-dimensional (3D) structure of the magnetic flux rope in the simulation domain after 2.5 hours of evolution with the gray plane showing photospheric vertical magnetic field $B_{z}$. Each of the two buoyant section rises and expands, bending the initial flux rope and forming an M-shaped configuration of magnetic fields extending from the convection zone into the corona. At the same time, the mass draining in the buoyant sections down along the field lines under gravitational force further enhances the buoyancy in the rising sections. Gravitational force of the heavy material keeps the central segment between the two rising sections down in the convection zone, while the two buoyant sections emerge. The downward push from the drained mass at the central segment between the rising bipoles maintains the configuration of multiplicity of bipoles in the domain, and prevents the multiple bipoles from merging into one pair of bipoles. Note that in the real solar convection zone, the downward convective flow may make an additional contribution to the downward push and facilitate the formation of multiple bipoles. The emerged sections expand dramatically due to the stratification of density in the surrounding plasma and especially when it approaches the photosphere, density drops by 6 orders of magnitude over a thin layer of $2 \mathrm{Mm}$, driving an enormous expansion of the flux rope in the horizontal direction. This expansion gives rise to an unstable situation where high-density material is supported by low-density plasma at the top of the flux rope. Under this condition, Rayleigh-Taylor instability takes place at the top boundary of the emerging fields and produces the undular structure on the field lines as represented by the red lines in Fig. 3. The grey plane of photospheric $B_{z}$ also shows strands of opposite polarities in the center of each bipole, manifesting the effect of Rayleigh-Taylor instability on the photospheric plane. Isobe et al. (2005, 2006) have shown that magnetic RayleighTaylor instability in simulations of flux emergence into preexisting coronal field also takes place in the top-heavy configuration caused by the expansion of the emerging dome and the draining of plasma along the field lines, and produces dense filaments mimicing $\mathrm{H} \alpha$ arch filaments, which may cause intermittent heating and the hot and cold loops observed in extreme ultraviolet images Yoshimura \& Kurokawa 1999).

The initial flux rope has a right-hand twist with $\mathrm{q}=0.5$, which causes the bipoles to initially emerge with its polarity inversion line at an angle to the axis of the flux rope. As the expansion of the buoyant emerging bipoles continues, the polarities in each bipole start to shift toward the central axis of the initial flux rope. At the center of the domain, the two polarities next to the dip in the M-shaped structure are pushed together by the expansion in each of the emerging bipoles, as well as the shifting motion toward the central axis, as shown in Fig. 4. These motions drive the two opposite polarities together to naturally form a sharp PIL between the the two polarities P1 and N2 (shown by arrows on Fig. 3) from the two bipoles. Note that underneath the photosphere, the two polarities P1 and N2 at the PIL are connected by a subsurface U-loop structure, while for each of the bipoles, i.e. N1-P1 and N2-P2, the opposite polarities are connected by $\Omega$-loops above the surface. At time $\mathrm{t}=3.5 \mathrm{hrs}$, the magnetic fields, shown by the red lines in Fig. 4 , expand further into the corona, forming overlying coronal loops connecting each of the bipoles. Importantly, along the PIL in the center, the magnetic fields from the two opposite polarities P1 and N2 are 
anti-parallel to each other, while their footpoints are pushed together during the emergence and expansion. In such a configuration of anti-parallel magnetic fields, strong current sheet builds up at the PIL where the two polarities P1 and N2 are moving toward each other, as shown by the magenta surfaces in Fig. 5 between the red lines connecting N1-P1 and N2-P2. With the continuing convergence of the two polarities during the expansion of the two emerging bipoles, current keeps thinning until reconnection takes places at the PIL, between the two nearby polarities P1 and N2. The three groups of lines in Fig. [ illustrate the complex coronal magnetic fields in our simulation domain. The reconnection forms the large-scale overlying loops connecting the two polarities at the two ends of the flux rope $\mathrm{N} 1$ and P2, as shown by the magenta lines in Fig. 6, as well as a set of small loops connecting the nearby polarities P1 and N2 in the center shown by the blue lines. The connectivity of the initial magnetic fields is colored by red, which shows an M-shaped structure. By comparing Fig. 4 and 6, we notice the change of the magnetic connectivity under the continuous expansion and emergence. We note that it is the reconnection at the PIL that produces the complexity in the structure of magnetic field. At this time, $\delta$-spot forms in the center with complex magnetic connectivity among the polarities and a compact magnetic structure at the PIL (see Fig. [5).

The evolution of total unsigned flux on the photosphere is shown in Fig. 7. We observe a fast growth in the total flux until about time $\mathrm{t}=2.6 \mathrm{hrs}$, when the two $\Omega$-shaped structures come into contact and collide. Until this time, the expansion and emergence of each structure are independent of each other and the magnetic flux simply grows into regions of less magnetic pressure, filling the space between the two buoyant segments rapidly. After time $t=2.6 \mathrm{hrs}$, the majority of the axial flux reaches the photosphere, as shown in Fig. 3, forming a quadupolar structure on the surface. After that, the growth of the total unsigned flux is slowed down, indicated by the less steep gradient from time $\mathrm{t}=2.6$ to $4.5 \mathrm{hrs}$, when the two polarities P1 and N2 collide into each other. During the collision, strong current builds up at the sharp PIL, and reconnection takes place between the two polarities P1 and N2, producing flux cancellation at the PIL. We evaluate the potential magnetic energy from the photospheric magnetogram and calculate total free magnetic energy in the corona by subtracting the potential energy from the magnetic energy in the coronal fields, as shown by the red dashed line in Fig. 7. The free energy keeps increasing with the flux emergence until 3.6 hrs, when the reconnection at the PIL starts to release the stored free magnetic energy by forming shorter loops shown by the blue lines in Fig. [6 with a total drop of $2.8 \times 10^{31} \mathrm{ergs}$ in free energy. The release of free energy is comparable with the estimated value of $3.4 \times 10^{31}$ ergs in AR 11158 as in Sun et al. (2012) although the energy release is more gradual in the simulation with no eruptions.

\section{Photospheric Flows and Energy Flux}

At the photosphere, the two buoyant sections of magnetic flux rope centered at $\mathrm{x}= \pm 15 \mathrm{Mm}$ first emerge as two separate bipoles at time $t=2.5 \mathrm{hrs}$, as shown in Panel (a) of Fig. 8. The distinct emergence of the two sections can also be viewed in the 3D magnetic structure shown at the same 
time in Fig. 3. Until then, the emergence of the two $\Omega$-shaped structures remains independant of each other, with only their footpoints being connected in the convection zone. The blue and yellow arrows represent the horizontal velocity in the positive and negative polarities, respectively. It is clear that at this time the velocity fields in N1-P1 and N2-P2 are mostly pointing outward from the center of each emerging section, representing the expansion of the emerging domes in a stratified atmosphere with dramatic drop in the pressure of the ambient plasma. Due to the initial seperation between the two density-depleted sections and the mass draining into the central dip, the two polarities P1 and N2 in the center remain separated at this time $\mathrm{t}=2.5 \mathrm{hrs}$ and unaffected by each other with a distance from each other as shown in Fig. 3. The continuous expansion during the emergence in the stratified atmosphere drives the outward motion of each polarities from the center of each bipole, as shown by the arrows representing horizontal velocity. Inevitably, the two $\Omega$-shaped emerging domes come into contact with each other and the two opposite polarities P1 and N2 are pushed together during the expansion, as shown by the velocity arrows in Panel (b) of Fig. 8. At the same time, a sharp PIL with high field gradient forms between P1 and N2 as they are pushed and squeezed into each other, which is found to be the preferential regions of flares and often used as an observational predictor of solar eruptive activities (Leka \& Barnes 2003; Schrijver 2007; Falconer et al. 2008; Mason \& Hoeksema 2010). More importantly, as the two polarities cannot annihilate with each other during the build up of sharp magnetic gradient, it is necessary that the two spots P1 and N2 take another curvilinear path around each other during the expansion of the two bipoles, which is also found in the observations of $\delta$-spots formed by collision of non-paired spots (Tang 1983). The horizontal velocity in the positive (P1) and negative (N2) polarities, as shown by the blue and yellow arrows, respectively, runs almost anti-parallel to each other along the PIL, exhibiting a strong shearing pattern between the two polarities. The velocity shear, driven by the spot motion (Zirin \& Liggett 1987) - flux emergence in our case, persists during the interaction of the two polarities P1 and N2, as shown by the anti-parallel pattern of velocity arrows in Panel (b)-(d) at the PIL. It streches the two sets of magnetic field lines connecting N1-P1 and N2-P2 respectively at the PIL in opposite directions and forms a set of highly sheared field lines at the interface between P1 and N2, as shown in Fig. 4. As discussed in Section 3, reconnection takes place at the PIL and forms a set of complex coronal structures as shown in Fig. 6. The reconnection here, similar to that in van Ballegooijen \& Mackay (2007) where two neigbouring $\Omega$-loops interact and reconnect at the PIL, releases the free magnetic energy by reconfiguring the stretched fields at the PIL into more relaxed ones. The continuous shearing motion represented by the arrows adjacent to the PIL both in Panel (d) of Fig. 8 and the movie available online then moves the footpoints of the post-reconnection low-lying field lines above the PIL in the opposite directions and stretch them into elongated and sheared magnetic structures. The highly sheared magnetic configuration at the PIL is believed to be one of the preceding signatures of great flares in $\delta$-spots (Zirin \& Liggett 1987), during which the free energy is released by relaxing and shortening the magnetic field lines.

Tang (1982) also pointed out that the the polarity axis in $\delta$-spots often deviates from Hale's law, based on sunspot observations from Mount Wilson. In our simulation, as shown by the evolution of polarities at the photopshere in Panel (a)-(d) of Fig. 8, we naturally get the inverted polarity of the 
$\delta$-spot P1 and N2 with the current configuration of the subsurface flux rope from the collision of the two emerging bipoles, as the following polarity of the $\delta$-spot is the leading one in the following bipole. During its evolution, however, the rotation motion of the $\delta$-spot drives the two polarities P1 and N2 in a direction towards obeying the Hale'slaw. The counterclockwise rotation of the $\delta$-spot is one of the manifestations of the curvilinear paths of the two polarities P1 and N2, which is ultimately driven by the expansion in each bipole.

As a result of the cuvilinear path of the spots, the magnetic fields between the two spots take the form of highly compressed and streched field lines at the PIL. The blue lines in Fig. 6 show a group of sheared reconnected fields among the more relaxed field lines connecting all four polarities in the domain shown by the red lines. Fig. 9 shows the strength of the horizontal magnetic field, i.e. $B_{h}=\left(B_{x}^{2}+B_{y}^{2}\right)^{1 / 2}$, in the $\delta$-spot region outlined by black rectangle in Fig. 8. Approaching the PIL, the horizontal field strength quickly increases to above $2 \mathrm{kG}$, as compared to the nearly vertical field in the center of each spot shown by the dark blue color. These strong transverse magnetic fields with strength comparable to that in the umbrae are confined to long, extremely narrow channels in $\delta$-spots in Fig. 9 and defined as "magnetic channels" by Zirin \& Wang (1993). In addition, the arrows in Fig. 9 representing the horizontal magnetic fields align almost parallel to the PIL, indicating that the transverse fields of kG-strength are along the PIL and thus highly non-potential (Low 1982), with the free magnetic energy stored which could possibly be released in eruptions.

Besides the shearing motion that forms highly elongated and sheared field lines adjacent to the PIL, a converging motion occurs in the outer periphery of the encountering spots, and keeps pushing the two spots P1 and N2 together. This converging motion results from the expansion and squeeze from the emerging bipoles of the writhed emerging rope, as shown by the 3D magnetic structure in Fig. 4. In Fig. 10, we calculated the Lorentz force in the $\delta$-spot region outlined by the rectangle in Panel (d) of Fig. 8, At the outer periphery of the $\delta$-spots P1 and N2 away from the PIL, we find that the horizontal Lorentz forces are pushing the two spots together with a converging pattern, consistent with the converging motion as shown in Panel (d) of Fig. 8. Meanwhile, at the PIL in the middle, apparently there is a strong repelling Lorentz force which pushes the two polarities in a direction away from each other. This repelling force naturally develops in such a system when two opposite polarities are pushed together with strong current sheet in between, while the converging motion in the $\delta$-spots is a manifestation of the expansion of each bipole. The combination of repelling force at the PIL and converging motion at the periphery gives the $\delta$-spots the observed curvilinear motion around one another. We here point out that this curvilinear motion is due to the emergence of the subsurface structure, which pushes and closely locks the two polarities $\mathrm{P} 1$ and $\mathrm{N} 2$ together during the expansion of the two bipoles. In van Ballegooijen \& Martens (1989), the presence of converging and shearing motions produces flux cancellation at the neutral line and the formation of a flux rope, which is not observed in our simulation here. The absence of such coherent a helical coronal flux rope may be the result of numerical diffusion (van Ballegooijen \& Mackay 2007), given the large cell size of $1237.4 \mathrm{~km}$ in the coronal region (see Section 2). 
To quantify the energy transfer associated with the horizontal and vertical motions of the magnetic fields, we calculate the Poynting flux associated with the two velocity components by

$$
\begin{aligned}
& F_{h}=-\frac{1}{8 \pi}\left(B_{x} u_{x}+B_{y} u_{y}\right) B z \\
& F_{v}=\frac{1}{8 \pi}\left(B_{x}^{2}+B_{y}^{2}\right) u_{z}
\end{aligned}
$$

Fig. 11] shows a zoom-in view of the distribution of the energy fluxes associated with horizontal (a) and vertical (b) motions, in the central polarities (same region as Fig. 10) at time $t=4: 40: 00$. The arrows in Panel (a) shows the horizontal flows in the $\delta$-spot and clearly represent a rotation pattern in each of the polarities as well as a strong shearing motion adjacent to the PIL shown by the white line. Significant energy flux into the corona occurs at the PIL, identified by the two red bands in Panel (a) which coincides with the shearing motion. In addition, within each of the polarity, the converging motion toward the PIL shown by the arrows also produces a strong energy flux into the corona. In Panel (b), the Poynting flux by the vertical motion of the fields also makes a great contribution to the energy transport into the corona, with brightennings along the PIL, indicating strong energy flux by the emerging of the magnetic fields. The energy flux concentrated at the PIL between the two colliding spots transports a great amount of free energy into magnetic fields above the PIL. As shown in Fig. 6, the magnetic fields are highly compressed and non-potential above the PIL, with a free energy of $8.3 \times 10^{31} \mathrm{ergs}$, about $46 \%$ of the total magnetic energy of $1.8 \times 10^{32}$ ergs. This stored free energy may be the energy source of the great flares that are often observed in $\delta$-spot regions (Zirin \& Liggett 1987).

\section{Summary and Conclusions}

In an active-region-scale domain, we carry out a simulation of the evolution of a twisted magnetic flux rope with two buoyant sections and study the formation of a compact $\delta$-spot like structure during the interaction of the two emerging sections as well as the maintenance of such compact structure with a realistic size scale. At the beginning, the two buoyant sections rise into the atmosphere independently of each other, expanding dramatically in a highly stratified background plasma. On the photosphere, the emergence of each section forms one pair of bipoles, each separated from the other. The separation in distance decreases as the two sections emerge and expand. The fact that the two buoyant sections originate from the same flux rope prevents the two bipoles from escaping from each other by subsurface connection(Toriumi et al. 2014). Inevitably the leading polarity of the following bipole collides with the following one of the leading bipole, forming a sharp PIL with a high magnetic gradient. The continuous expansion during the emergence keeps pushing together the two polarities in the center and locks the two spots closely, producing a compact $\delta$-spot active region on the photosphere. The two colliding polarities take a curvilinear path around one another, producing the observed shearing and rotation motion. At the PIL, the horizontal velocity in the two polarities runs anti-parallel with each other. The magnetic fields is highly compressed and almost parallel to PIL in the middle of the $\delta$-spot. The sheared transverse magnetic field of $\mathrm{kG}$ 
strength possesses a significant amount of free magnetic energy up to $9.3 \times 10^{31} \mathrm{ergs}$, as a potential source for eruptions and flares that are often associated with $\delta$-spot active regions. The $\delta$-spot in such an active-region-scale simulation produces detailed dynamics comparable with observations (Tang 1983), such as the inverted polarity against Hale's law, sharp PIL with sheared kG transverse magnetic fields, the curvilinear motion of the polarities, as well as the complex coronal magnetic connectivity.

As collision of two bipolar groups is the most popular type of formation of $\delta$-spots (Zirin \& Liggett 1987), it is crucial to understand mechanisms that closly lock the two bipoles together during their emergence and expansion. As has been pointed out by Toriumi et al. (2014), the subsurface configuration plays an important role in the formation of a compact photospheric magnetic structure. The two emerging bipoles in our simulation, deeply rooted and connected together in the convection zone, are not able to separate from each other due to the internal linkage. The emergence and expansion drive the two spots into a curvilinear path around one another which manifests as rotation of the spots and shearing motion at the PIL on the photosphere. Nevertheless, it is the repelling force from each $\delta$-spot's bipolar counterpart that pushes the two polarities in the $\delta$-spot together and maintains the integrity of the quadupolar structure during the evolution. In the realistic solar convection zone, the subsurface configuration of multiple buoyantly emerging sections on the same flux rope may occur when the flux rope encounters multiple cells of upflowing plasma, which is possible in the turbulent interior (Nelson et al. 2011; Fan \& Fang 2014). Realistic simulations with convective motions (Rempel \& Schlichenmaier 2011; Fang et al. 2012b) have to be implemented to study the detailed dynamics in the emergence from the interior to the photosphere. In addition, in simulations with the effect of Coriolis force on the flux emergence, a retrograde flow naturally develops along the flux rope and produces assymetry in the two polarities of each emerging loop, with the leading one being more dominant (Fan et al. 1993; Rempel \& Cheung 2014). Therefore, in the process where a $\delta$-spot is formed by collison of two bipoles, the fact that the following polarity in the $\delta$-spot is the leading polarity of the following bipole could also explain the dominance of the following polarity oftern observed in such $\delta$-spots (Tang 1983).

We thank Anna Malanushenko and the referee for constructive comments and discussions. The work here is supported by NASA LWSCSW grant NNX13AJ04A. The National Center for Atmospheric Research (NCAR) is sponsored by the National Science Foundation. The simulations described here were carried out on the Stampede Supercomputer in the Texas Advanced Computing Center (TACC) at the University of Taxas at Austin and and Yellowstone Supercomputer at NCAR. 


\section{REFERENCES}

Falconer, D. A., Moore, R. L., \& Gary, G. A. 2008, ApJ, 689, 1433

Fan, Y. 2001, ApJ, 554, L111

Fan, Y. \& Fang, F. 2014, ApJ, 789, 35

Fan, Y., Fisher, G. H., \& Deluca, E. E. 1993, ApJ, 405, 390

Fan, Y., Zweibel, E. G., Linton, M. G., \& Fisher, G. H. 1999, ApJ, 521, 460

Fang, F., Manchester, IV, W., Abbett, W. P., \& van der Holst, B. 2012a, ApJ, 754, 15

—. 2012b, ApJ, 745, 37

Hagyard, M. J., Teuber, D., West, E. A., \& Smith, J. B. 1984, Sol. Phys., 91, 115

Isobe, H., Miyagoshi, T., Shibata, K., \& Yokoyama, T. 2005, Nature, 434, 478

—. 2006, PASJ, 58, 423

Leka, K. D. 1997, ApJ, 484, 900

Leka, K. D. \& Barnes, G. 2003, ApJ, 595, 1277

Leka, K. D., Canfield, R. C., McClymont, A. N., \& van Driel-Gesztelyi, L. 1996, ApJ, 462, 547

Linton, M. G., Dahlburg, R. B., Fisher, G. H., \& Longcope, D. W. 1998, ApJ, 507, 404

Linton, M. G., Fisher, G. H., Dahlburg, R. B., \& Fan, Y. 1999, ApJ, 522, 1190

Lites, B. W., Low, B. C., Martinez Pillet, V., Seagraves, P., Skumanich, A., Frank, Z. A., Shine, R. A., \& Tsuneta, S. 1995, ApJ, 446, 877

Liu, Y. \& Zhang, H. 2001, A\&A, 372, 1019

Low, B. C. 1982, Sol. Phys., 77, 43

Mason, J. P. \& Hoeksema, J. T. 2010, ApJ, 723, 634

Nelson, N. J., Brown, B. P., Brun, A. S., Miesch, M. S., \& Toomre, J. 2011, ApJ, 739, L38

Patty, S. R. \& Hagyard, M. J. 1986, Sol. Phys., 103, 111

Powell, K. G., Roe, P. L., Linde, T. J., Gombosi, T. I., \& de Zeeuw, D. L. 1999, Journal of Computational Physics, 154, 284

Rempel, M. \& Cheung, M. C. M. 2014, ApJ, 785, 90 
Rempel, M. \& Schlichenmaier, R. 2011, Living Reviews in Solar Physics, 8, 3

Sammis, I., Tang, F., \& Zirin, H. 2000, ApJ, 540, 583

Schmieder, B., Hagyard, M. J., Guoxiang, A., Hongqi, Z., Kalman, B., Gyori, L., Rompolt, B., Demoulin, P., \& Machado, M. E. 1994, Sol. Phys., 150, 199

Schrijver, C. J. 2007, ApJ, 655, L117

Sun, X., Hoeksema, J. T., Liu, Y., Wiegelmann, T., Hayashi, K., Chen, Q., \& Thalmann, J. 2012, ApJ, 748, 77

Tanaka, K. 1991, Sol. Phys., 136, 133

Tang, F. 1982, Sol. Phys., 75, 179

—. 1983, Sol. Phys., 89, 43

Toriumi, S., Iida, Y., Kusano, K., Bamba, Y., \& Imada, S. 2014, Sol. Phys., 289, 3351

Tóth, G., van der Holst, B., Sokolov, I. V., De Zeeuw, D. L., Gombosi, T. I., Fang, F., Manchester, W. B., Meng, X., Najib, D., Powell, K. G., Stout, Q. F., Glocer, A., Ma, Y.-J., \& Opher, M. 2012, Journal of Computational Physics, 231, 870

van Ballegooijen, A. A. \& Mackay, D. H. 2007, ApJ, 659, 1713

van Ballegooijen, A. A. \& Martens, P. C. H. 1989, ApJ, 343, 971

Yoshimura, K. \& Kurokawa, H. 1999, ApJ, 517, 964

Zirin, H. \& Liggett, M. A. 1987, Sol. Phys., 113, 267

Zirin, H. \& Wang, H. 1993, Sol. Phys., 144, 37

This preprint was prepared with the AAS $\mathrm{LAT}_{\mathrm{E}} \mathrm{X}$ macros v5.0. 


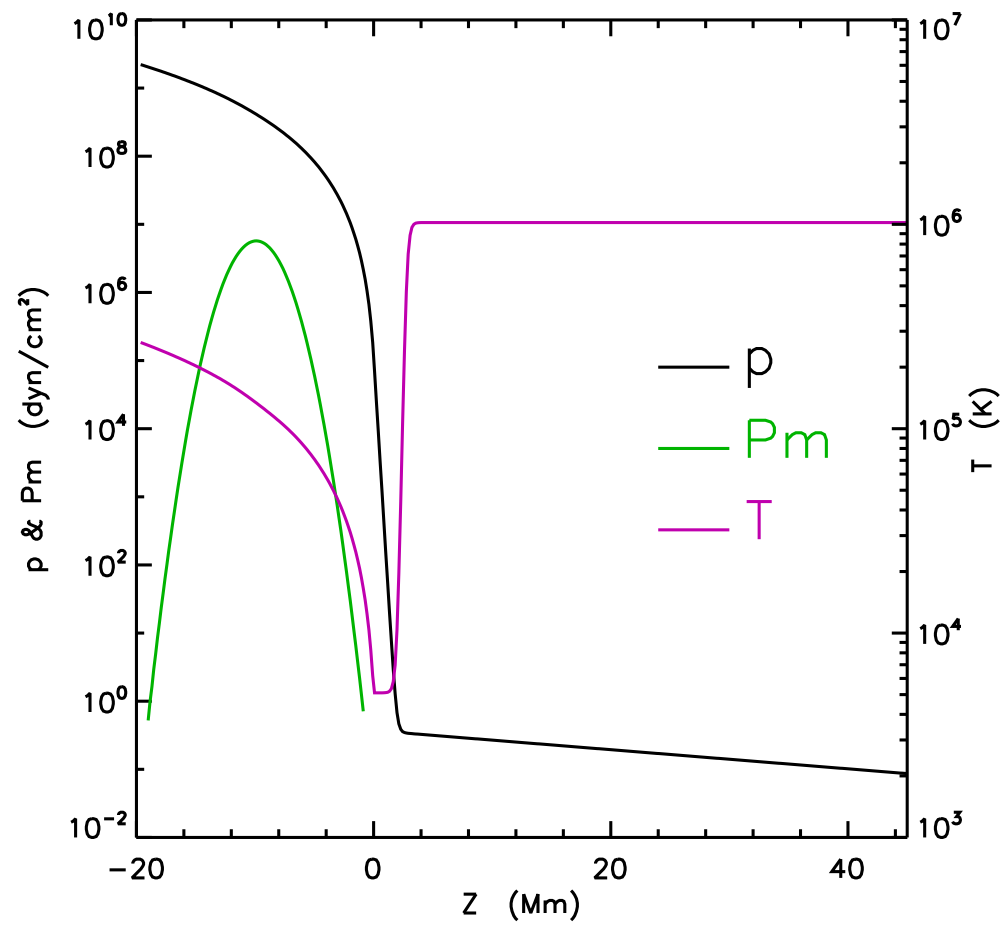

Fig. 1. - Initial vertical stratification of pressure (black) and temperature (purple) in the domain. The green line shows the distribution of magnetic pressure in a cross-section cut of the flux rope.

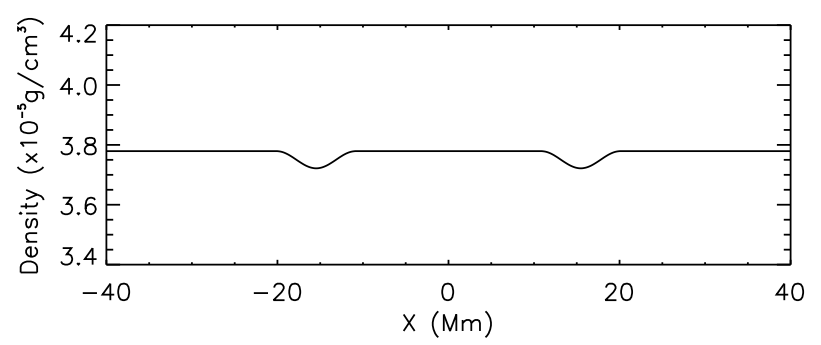

Fig. 2.- Distribution of the initial density along the axis of the flux rope with two buoyant sections centered at $\mathrm{x}= \pm 15 \mathrm{Mm}$. 


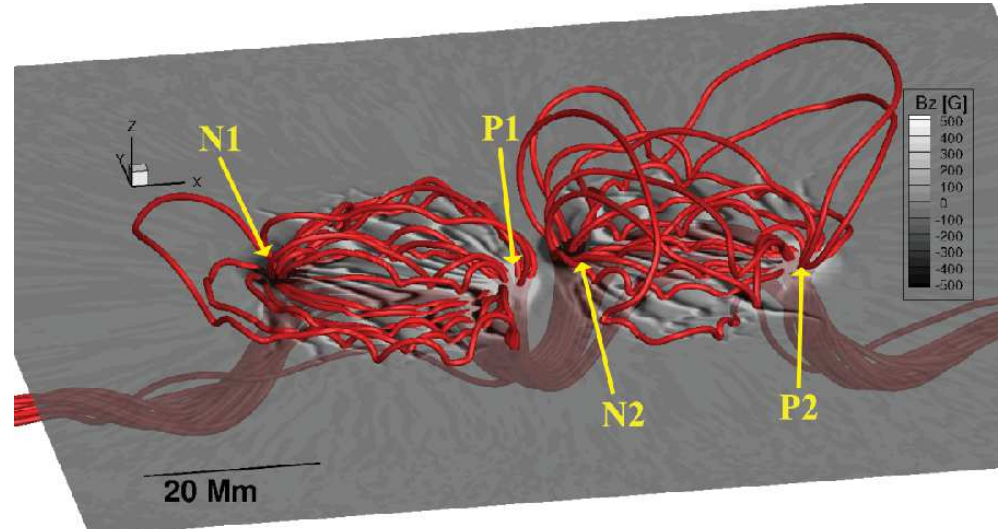

Fig. 3. - 3D structure of the M-shaped magnetic field lines (red lines) in the domain at time $\mathrm{t}=$ 2.5 hrs. The plane shows the photospheric magnetogram.

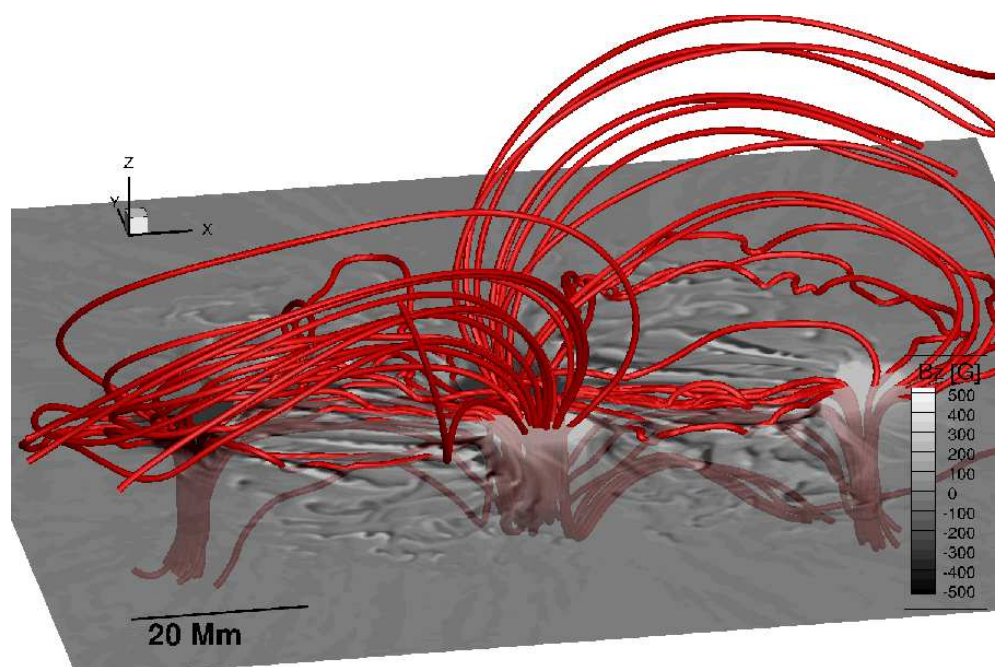

Fig. 4. - Same as Fig. 3 but at time $t=3.5$ hrs. 


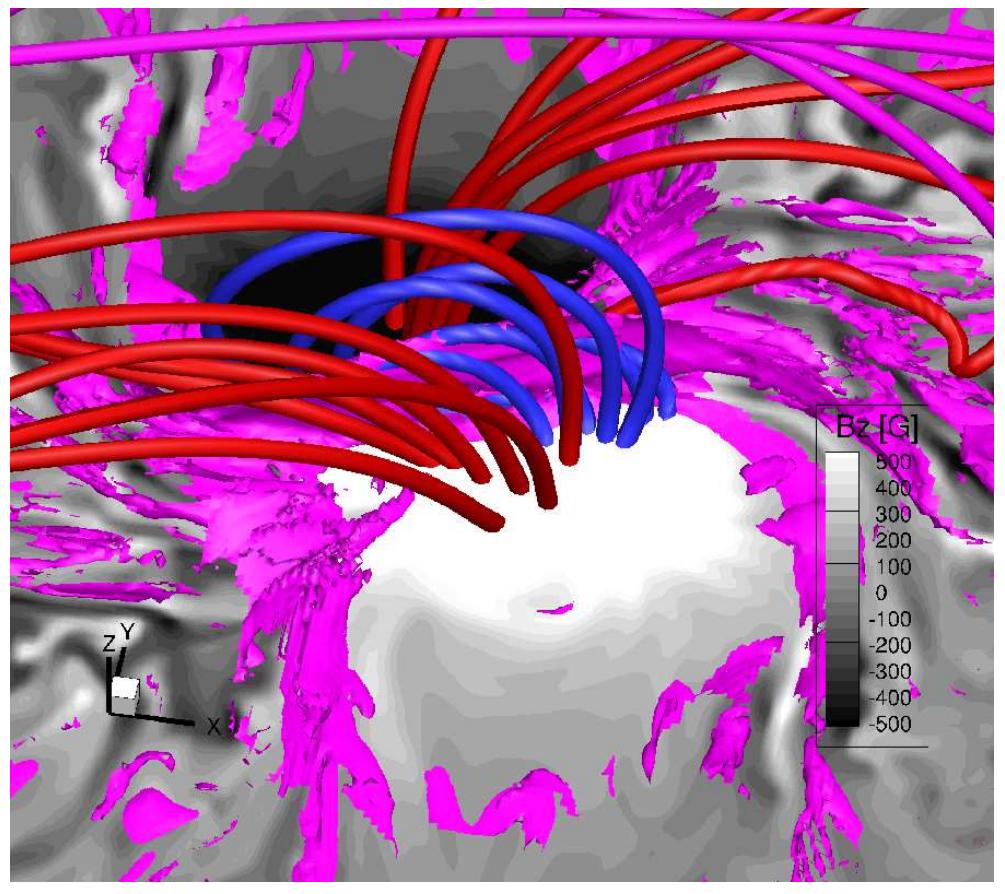

Fig. 5.- The $\delta$-spot region at time $\mathrm{t}=4.5 \mathrm{hrs}$ with magenta isosurfaces of $|J| /|B|=100$ in regions of $\mathrm{B}_{h}>300 \mathrm{G}$ and colored lines representing the magnetic field lines.

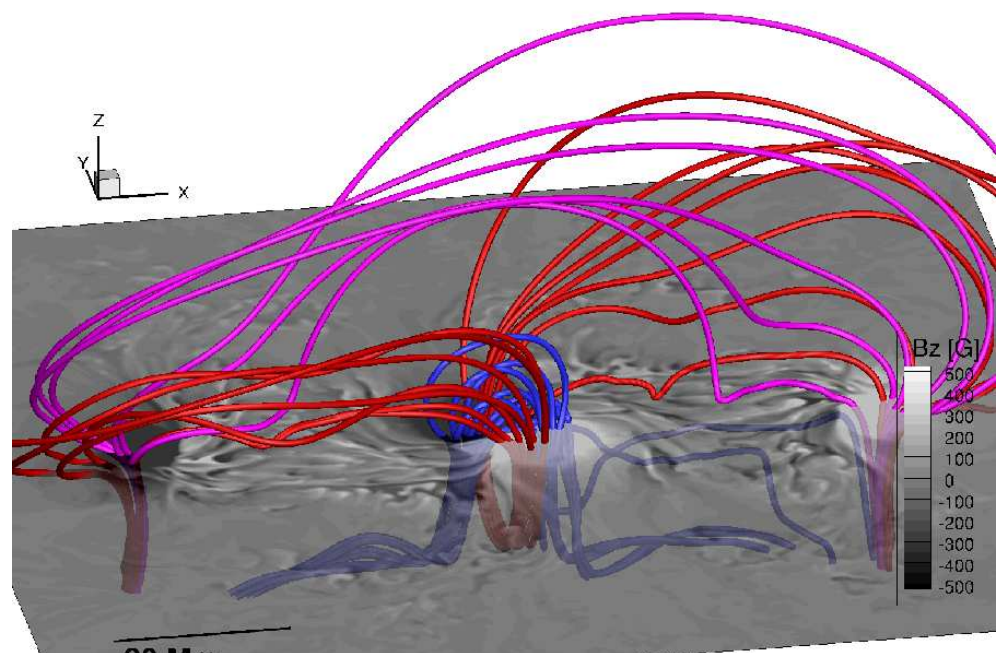

$20 \mathrm{Mm}$

Fig. 6.- Same as Fig. 3 but at time $\mathrm{t}=4.5 \mathrm{hrs}$. The magenta and blue lines are showing the overlying and low-lying magnetic field lines after the reconnection, respectively. The red lines show the field lines connecting the four polarities. 


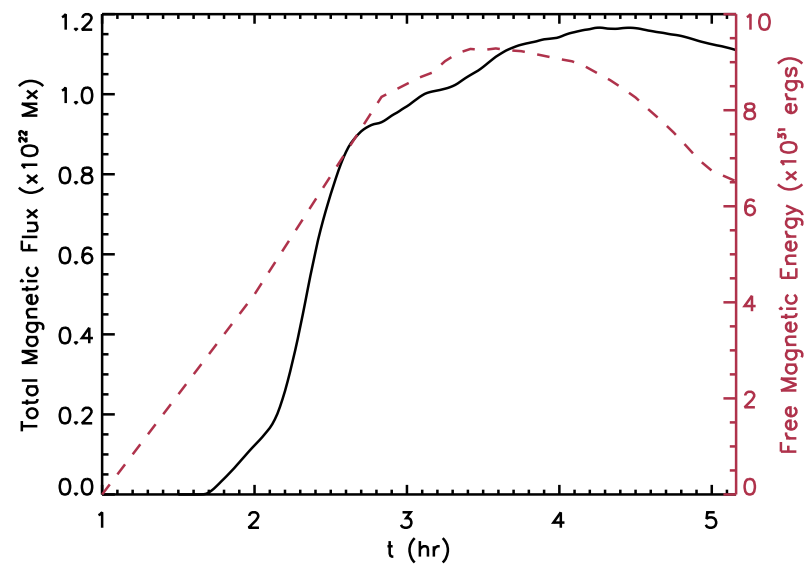

Fig. 7.- Evolution of total unsigned magnetic flux at the photosphere in the computational domain and the coronal free magnetic energy. 


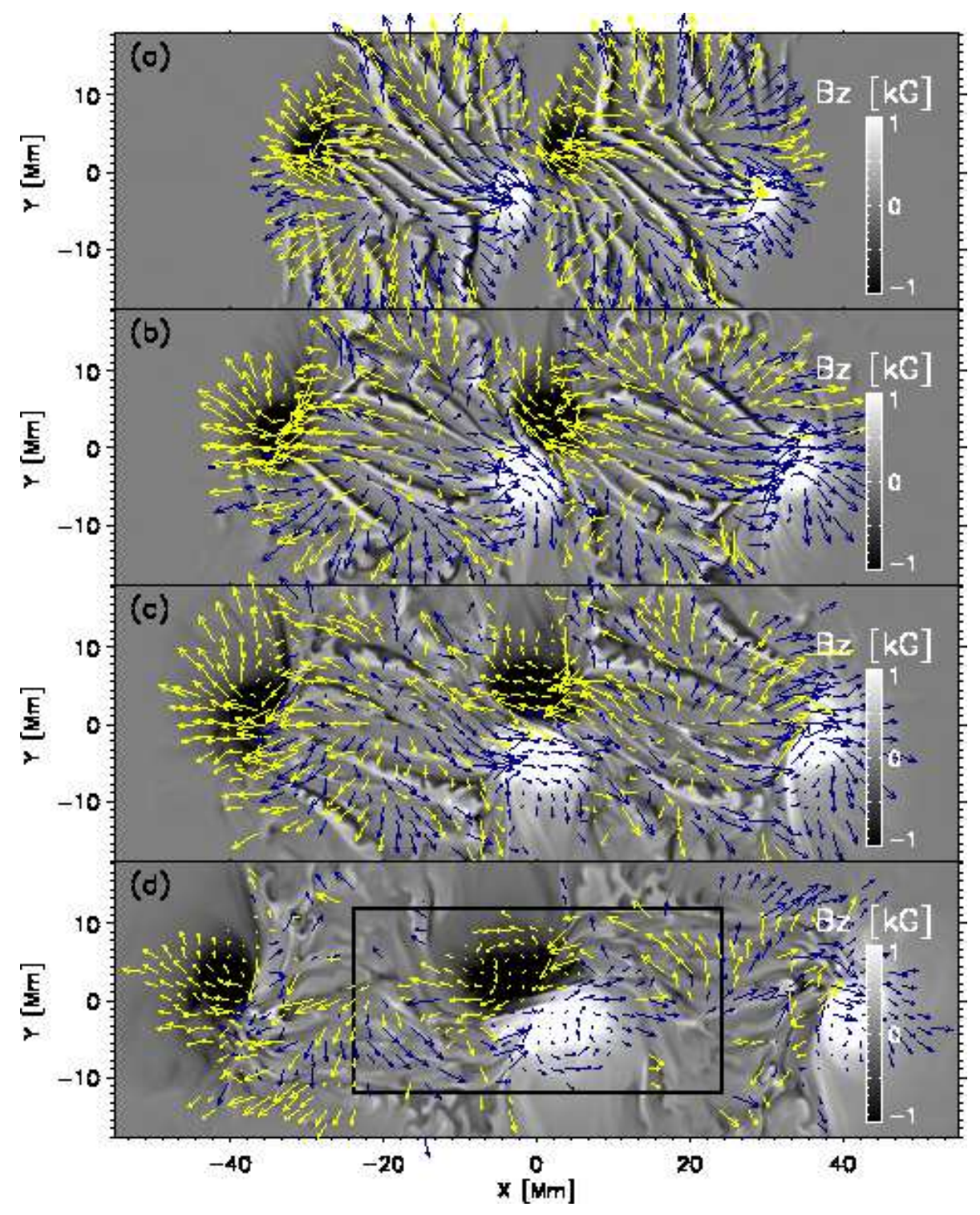

Fig. 8. - Magnetogram at time $t=2.5$ (a), 3.0 (b), 3.5 (c) and 4.5 (d) hrs. Blue and yellow arrows show the horizontal velocity in positive and negative polarities with $\left|B_{z}\right|>800 \mathrm{G}$, respectively. A movie showing the evolution of magnetogram with horizontal velocities is available online. 


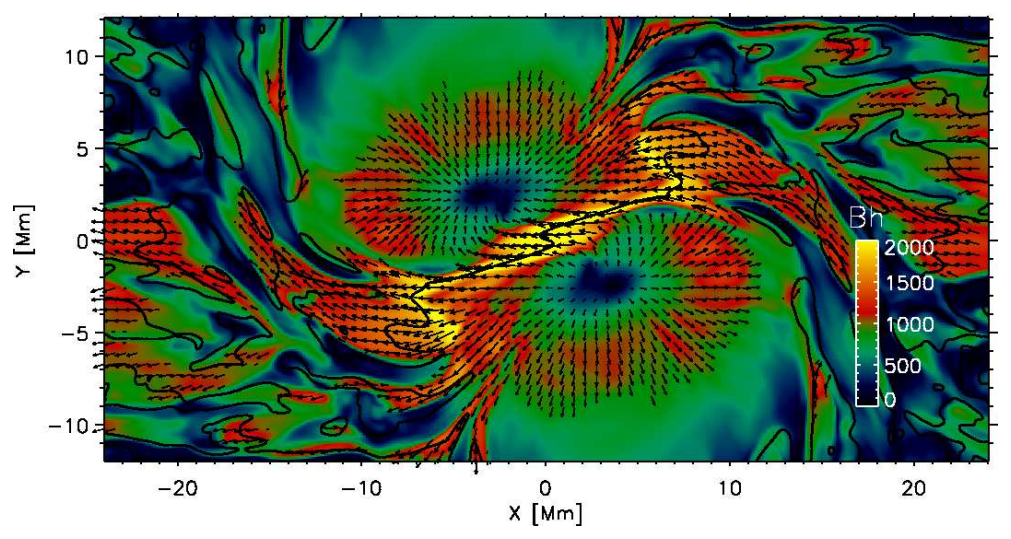

Fig. 9.- The horizontal magnetic field with color showing strength and arrows showing directions in the area outlined by the rectangle in Panel $(\mathrm{d})$ of Fig. 8 at time $\mathrm{t}=4.5 \mathrm{hrs}$. The black lines represent the PILs.

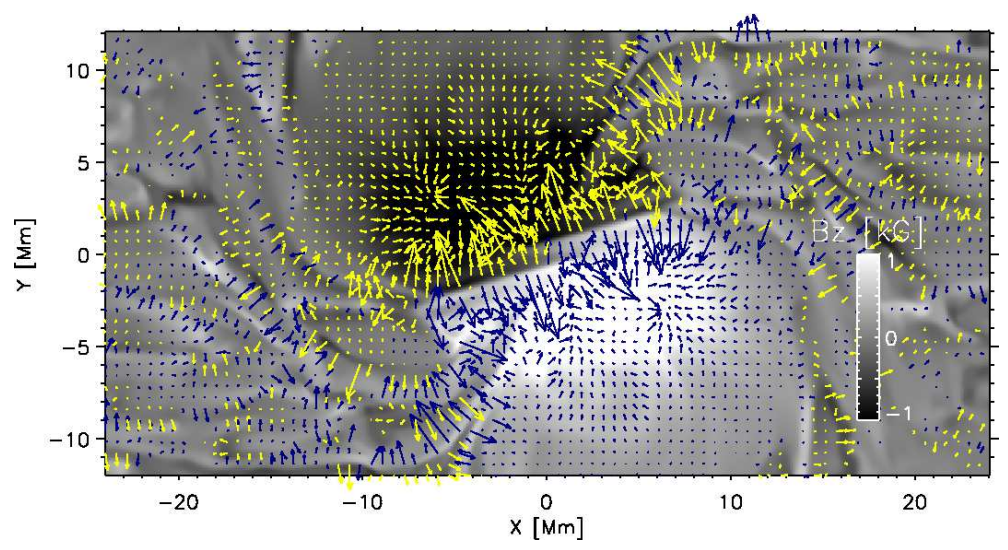

Fig. 10. - Vertical magnetic field $\mathrm{B}_{z}$ in the area outlined by the rectangle in Panel (d) of Fig. 8 at time $\mathrm{t}=4.5 \mathrm{hrs}$ with blue and yellow arrows showing the horizontal Lorentz force in positive and negative polarities, respectively. 

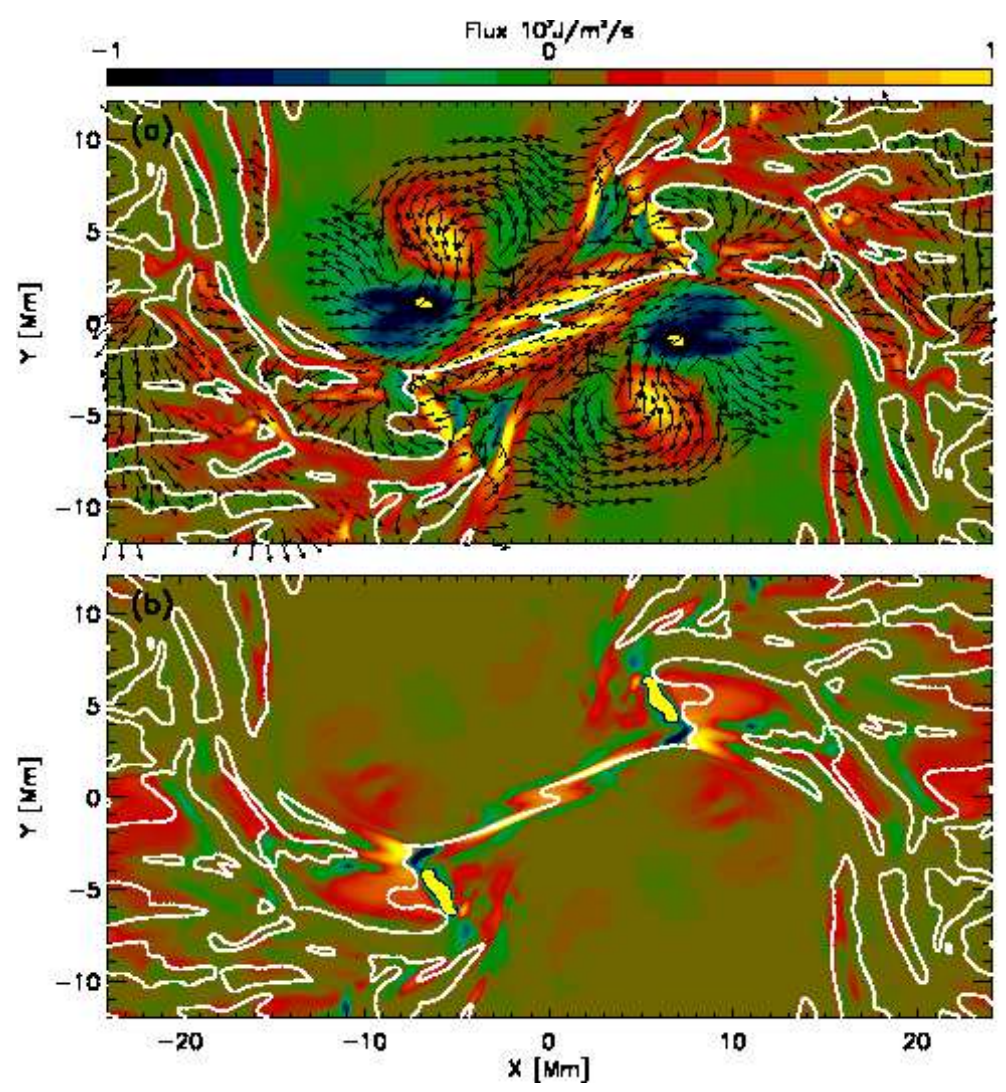

Fig. 11. - Poynting flux associated with the horizontal (a) and vertical (b) motions of the magnetic field lines at time $\mathrm{t}=4: 40: 00$. The black arrows represent the horizontal flows in regions with $\left|B_{z}\right|>$ $800 \mathrm{G}$ and white lines show the PIL. 